Research article

Open Access

\title{
Metastasis of hormone-independent breast cancer to lung and bone is decreased by $\alpha$-difluoromethylornithine treatment
}

\author{
Monica M Richert1, Pushkar A Phadke1, Gail Matters², Douglas J DiGirolamo1, \\ Sharlene Washington ${ }^{3}$, Laurence M Demers ${ }^{4}$, Judith S Bond ${ }^{2}$, Andrea Manni ${ }^{3}$ and \\ Danny R Welch ${ }^{1}$
}

\author{
1Department of Pathology, Comprehensive Cancer Center, Center for Metabolic Bone Disease, National Foundation for Cancer Research - Center \\ for Metastasis Research, University of Alabama at Birmingham, Alabama, USA \\ 2Department of Biochemistry and Molecular Biology, The Pennsylvania State University College of Medicine, Hershey, Pennsylvania, USA \\ ${ }^{3}$ Department of Medicine, The Pennsylvania State University College of Medicine, Hershey, Pennsylvania, USA \\ ${ }^{4}$ Department of Pathology, The Pennsylvania State University College of Medicine, Hershey, Pennsylvania, USA
}

Corresponding author: Monica M Richert, mrichert@path.uab.edu

Received: 5 May 2005 Revisions requested: 21 Jun 2005 Revisions received: 22 Jun 2005 Accepted: 1 Jul 2005 Published: 9 Aug 2005

Breast Cancer Research 2005, 7:R819-R827 (DOI 10.1186/bcr1292)

This article is online at: http://breast-cancer-research.com/content/7/5/R819

(c) 2005 Richert et al, licensee BioMed Central Ltd.

This is an Open Access article distributed under the terms of the Creative Commons Attribution License (http://creativecommons.org/licenses/by/ 2.0), which permits unrestricted use, distribution, and reproduction in any medium, provided the original work is cited.

\begin{abstract}
Introduction Polyamines affect proliferation, differentiation, migration and apoptosis of cells, indicating their potential as a target for cancer chemotherapy. Ornithine decarboxylase converts ornithine to putrescine and is the rate-limiting step in polyamine synthesis.

$\alpha$-Difluoromethylornithine (DFMO) irreversibly inhibits ornithine decarboxylase and MDA-MB-435 human breast cancer metastasis to the lung without blocking orthotopic tumor growth. This study tested the effects of DFMO on orthotopic tumor growth and lung colonization of another breast cancer cell line (MDA-MB-231) and the effects on bone metastasis of MDAMB-435 cells.

Methods MDA-MB-231 cells were injected into the mammary fat pad of athymic mice. DFMO treatment ( $2 \%$ per orally) began at the day of tumor cell injection or 21 days post injection. Tumor growth was measured weekly. MDA-MB-231 cells were injected into the tail vein of athymic mice. DFMO treatment began 7 days prior to injection, or 7 or 14 days post injection. The number and incidence of lung metastases were determined. Green fluorescent protein-tagged MDA-MB-435 cells were injected into the left cardiac ventricle in order to assess the incidence and extent of metastasis to the femur. DFMO treatment began 7 days prior to injection.
\end{abstract}

Results DFMO treatment delayed MDA-MB-231 orthotopic tumor growth to a greater extent than growth of MDA-MB-435 tumors. The most substantial effect on lung colonization by MDA-MB-231 cells occurred when DFMO treatment began 7 days before intravenous injection of tumor cells (incidence decreased $28 \%$ and number of metastases per lung decreased 35-40\%). When DFMO treatment began 7 days post injection, the incidence and number of metastases decreased less than $10 \%$. Surprisingly, treatment initiated 14 days after tumor cell inoculation resulted in a nearly $50 \%$ reduction in the number of lung metastases without diminishing the incidence. After intracardiac injection, DFMO treatment decreased the incidence of bone metastases (55\% vs $87 \%$ ) and the area occupied by the tumor $\left(1.66 \mathrm{~mm}^{2}\right.$ vs $\left.4.51 \mathrm{~mm}^{2}, P<0.05\right)$.

Conclusion Taken together, these data demonstrate that DFMO exerts an anti-metastatic effect in more than one hormone-independent breast cancer, for which no standard form of biologically-based treatment exists. Importantly, the data show that DFMO is effective against metastasis to multiple sites and that treatment is generally more effective when administered early. 


\section{Introduction}

Metastasis is the leading cause of death of breast cancer patients. As tumors progress from hormone-dependent to hormone-independent, their risk of metastasis increases and the treatment options decrease. There are currently no efficacious biologically-based therapies for the more aggressive hormoneindependent breast cancers. It is therefore important to explore potential therapies that may be effective against both metastasis and growth of hormone-independent breast tumors.

Polyamines are organic compounds derived from decarboxylation of the amino acid ornithine. These compounds play a role in a wide variety of cellular functions including proliferation, differentiation, migration and apoptosis [1]. They are required for cell viability, as demonstrated by the lethality of ornithine decarboxylase (ODC) null mutations in mice [2]. ODC is the rate-limiting enzyme in polyamine synthesis: It converts ornithine to putrescine to begin the polyamine cascade. Overexpression of ODC in the immortalized, but non-tumorigenic, mammary epithelial cell line MCF10A results in a partially transformed phenotype as well as in the activation of ERK, which is often activated in breast cancers [3,4]. Previous studies demonstrated that increased ODC activity correlates with transition to hormone independence, increased anaplasia and metastasis. Increased ODC activity is an independent adverse prognostic factor for overall breast cancer survival in women with localized disease [5-7], suggesting that increased activity of the polyamine pathway may result in more aggressive, hormone-independent breast cancers.

$\alpha$-Difluromethylornithine (DFMO) is an irreversible inhibitor of ODC. Treatment with DFMO results in decreased polyamine pools causing a cytostatic effect. Our laboratory previously demonstrated that DFMO treatment decreased lung metastasis from the estrogen receptor-negative/progesterone receptor-negative breast carcinoma cell line MDA-MB-435 xenografts, while having only a modest or absent effect on the growth of the orthotopic tumors [8,9]. Previous studies have shown that DFMO (0.5-3\% in drinking water) was well tolerated in both rats and mice. The minimal toxicities of DFMO make it a suitable candidate for long-term treatments.

The studies described here were designed to address two questions. First, does DFMO reduce metastasis to sites other than lung? To address this question, we took advantage of a recently developed enhanced green fluorescent protein (GFP)-expressing variant of MDA-MB-435 [10,11]. Second, does DFMO affect orthotopic tumor growth and metastasis of another hormone-independent breast carcinoma? To test this question, we utilized the estrogen receptor-negative/progesterone receptor-negative MDA-MB-231 cell line. We present here that DFMO can decrease metastasis of MDA-MB-435 cells to the bone and can affect lung metastasis and orthotopic tumor growth of MDA-MB-231 cells.

\section{Materials and methods Cell lines}

MDA-MB-231 and MDA-MB-435 human breast cancer cell lines were kindly provided by Dr Janet E Price at the University of Texas MD Anderson Cancer Center. They were cultured in DMEM/Ham's F12 medium supplemented with 5\% fetal bovine serum, $1 \%$ non-essential amino acids, $1 \mathrm{mM}$ sodium pyruvate, and were maintained at $37^{\circ} \mathrm{C}$ with $5 \% \mathrm{CO}_{2}$ in a humidified atmosphere. The cells were passaged using $0.125 \%$ trypsin, $2 \mathrm{mM}$ ethylenediamine tetraacetic acid in $\mathrm{Ca}^{2+} / \mathrm{Mg}^{2+}$-free Dulbecco's PBS.

\section{Metastasis assays}

All animals were maintained under the guidelines of the IACUC of the University of Alabama at Birmingham under registered protocols. Female athymic mice (Harlan Sprague-Dawley, Inc., Indianapolis, IN, USA) were used for all studies.

To study bone metastasis, 4-week-old to 6-week-old female mice were injected intracardially with $3 \times 10^{5}$ GFP-labeled MDA-MB-435 cells in $0.2 \mathrm{ml}$ ice-cold Hanks Balanced Salt Solution (HBSS). Seven days prior to injection, two groups of 10 mice each were randomly selected. One group remained untreated, while the second group was provided DFMO as a $2 \%$ solution in the drinking water ad libitum until 6 weeks post injection. This dose had been previously demonstrated as efficacious. The mice were euthanized and the femurs dissected away from the soft tissue. Bone metastases were visualized by fluorescence microscopy [10,11]. Brightfield and fluorescent photographs were analyzed using Sigma Scan (SPSS Inc., Chicago, IL, USA) to determine the total area of bone, the area of bone occupied by fluorescing tumor cells and the percentage of bone occupied by fluorescing tumor cells.

MDA-MB-231 breast cancer cells $\left(2 \times 10^{5}\right.$ cells in $0.2 \mathrm{ml}$ HBSS) were injected intravenously into the lateral tail vein of 3-week-old to 4-week-old athymic mice to evaluate lung colonization as metastases from an orthotopically growing tumor are relatively rare from this cell line. Each treatment group consisted of 20 mice. DFMO treatment ( $2 \%$ in drinking water) was administered using three schedules beginning: 7 days prior to tumor cell injection, 7 days after tumor cell injection, or 14 days after tumor cell injection. DFMO was administered until the experiment was terminated (4-6 weeks post injection). Control animals did not receive DFMO. At termination, the lungs were removed and fixed in Bouin's fixative diluted 1:5 with neutral-buffered formalin. The number of lungs with surface metastases were determined, as well as the number of surface metastases per lung by examination under a dissecting microscope, as described elsewhere [12].

Orthotopic tumor growth was measured by injecting MDAMB-231 breast cancer cells $\left(5 \times 10^{5}\right.$ cells in $\left.0.1 \mathrm{ml} \mathrm{HBSS}\right)$ into the second thoracic mammary fat pad of 5-week-old to 6 week-old female athymic nude mice, as described previously 
[12]. DFMO treatment ( $2 \%$ in drinking water) began at the time of mammary fat pad injection or at 21 days post injection. The control group consisted of 17 mice, the group started on DFMO at the time of injection consisted of 20 mice, while three mice began treatment 21 days post injection. Tumor growth was monitored weekly by measuring the tumor length and width with a caliper and was reported as the mean tumor diameter as previously described [12]. Since DFMO retarded tumor growth, DFMO-treated mice were euthanized when average local tumor diameters reached approximately $1.5 \mathrm{~cm}$. In a subsequent experiment, all animals were euthanized 6 weeks post tumor cell injection. To examine growth parameters, mice were injected with $100 \mathrm{mg} / \mathrm{kg}$ BrdU approximately 2 hours prior to euthanasia. Tumors were removed and divided into two portions. One aliquot was fixed in 10\% neutral-buffered formalin and processed for histological analysis. The other aliquot was frozen in liquid nitrogen and stored at $-70^{\circ} \mathrm{C}$ for analysis of polyamine levels as described $[9,13]$.

\section{Polyamine levels}

Athymic mice were injected subcutaneously (or in the mammary fat pad as already described) with $5 \times 10^{5}$ MDA-MB-231 cells in $0.1 \mathrm{ml} \mathrm{HBSS}$. The comparison of orthotopic sites with ectopic sites was carried out in order to evaluate potential pharmacologic differences. The mice were separated into two animals per group. The control group was left untreated, while DFMO treatment began 7 days prior to tumor cell injection, on the day of injection, or 21 days post tumor cell injection. At 6 weeks post injection, tumors were removed and homogenized in $25 \mathrm{mM}$ Tris- $\mathrm{HCl}$ buffer, $\mathrm{pH} 7.4$, containing $0.1 \mathrm{mM}$ ethylenediamine tetraacetic acid and $2.5 \mathrm{mM}$ dithiothreitol. The homogenates were centrifuged for $30 \mathrm{~min}$ at $20,000 \times \mathrm{g}$. An aliquot of the supernatant was used to measure ODC activity. The remaining aliquot was extracted with $0.6 \mathrm{~N}$ perchloric acid for 1 hour at $4^{\circ} \mathrm{C}$ prior to centrifugation at $15,000 \times g$ for 15 $\mathrm{min}$. The supernatant was used for polyamine determination. Polyamine levels were determined using high-pressure liquid chromatography as previously described $[9,13]$.

\section{Quantification of bromodeoxyuridine incorporation}

Incorporation of BrdU was determined using immunohistochemical staining. Briefly, the sections were deparaffinized using xylenes and ethanol, and were then treated with $3 \%$ hydrogen peroxide for $10 \mathrm{~min}, 0.1 \%$ trypsin for $30 \mathrm{~min}$ and 2 $\mathrm{N} \mathrm{HCl}$ for 30 min with PBS washes between each treatment. The sections were blocked with $5 \%$ normal goat serum in $1 \%$ BSA, $0.05 \%$ Tween 20 and $0.1 \% \mathrm{NaN}^{3}$ in PBS followed by a 2-hour incubation at $37^{\circ} \mathrm{C}$ in a $1: 1000$ dilution of a monoclonal anti-BrdU antibody (B2531; Sigma, St. Louis, MO, USA). The sections were rinsed and then incubated for 1 hour at $37^{\circ} \mathrm{C}$ in a 1:5000 dilution of anti-mouse AlexaFluor Green. After the final washes, the sections were cover-slipped in Vectashield mounting media containing DAPI diluted $1: 7$ with non-DAPIcontaining Vectashield mounting media. Five photographs $(100 \times$ magnification) from two sections of each tumor were counted for BrdU-labeled cells and the total number of labeled cells was graphed.

\section{Quantification of TUNEL staining}

Apoptotic cells were identified using the Apoptag plus staining kit from Chemicon (S7101; Temecula, CA, USA) according to the manufacturer's instructions. Five photographs $(63 \times$ magnification) from two sections of each tumor were counted for TUNEL-labeled cells, and the total number of labeled cells was graphed.

\section{Semi-quantitative RT-PCR}

Total RNA was isolated using Trizol (Invitrogen, Carlsbad, CA, USA) and $500 \mathrm{ng}$ was used for RT-PCR with a Superscriptlll/ Platinum Taq One Step Kit (Invitrogen) as directed. Human glyceraldehyde-3-phosphate dehydrogenase primers (5'GTGAAGGTCGGAGTCAACGGATT-3' and 5'-AGTGATGGCATGGACTGTGGTC-3') and hypoxanthine phosphoribosyl transferase primers (5'CCAAAGATGGTCAAGGTCGC-3' and 5'-CTGCTGACAAAGATTCACTGG-3') were used to assess equal template loadings, and the linear range for each primer pair was determined independently. Human meprin $\alpha$ levels were determined with the following primers: 5'-ATCGGAGGCACGGCTGGCGTG-3' and 5'GCCTGCCCTCATGGAGCTTACAG-3'. RT-PCR products were separated on a $1 \%$ TAE/agarose gel and quantified using a Stratagene Eagle Eye system with multiple integrations.

\section{Statistics}

Comparisons between treatment groups and control-treated mice were made using SigmaStat statistical software (SPSS Inc.). For multiple group comparisons, one-way analysis of variance was performed, followed by the Student-NeumannKeuls post-test. Results were considered statistically different if $P<0.05$.

\section{Results and discussion}

In normal tissues, ODC activity is increased by a variety of environmental and genetic factors associated with carcinogenesis, including ultraviolet light, asbestos and androgens. Increased ODC activity persists in and is associated with a wide variety of epithelial neoplasms including breast, skin, colon and prostate (reviewed in [1]). Together, these correlations suggest a role for ODC in tumor development and progression. Functional evidence supports this conclusion. In skin carcinogenesis models, ODC heterozygous knockout mice have significantly reduced tumor development [14].

Hormone-independent breast cancers are generally more aggressive and metastatic than hormone-responsive tumors. Unfortunately, there are currently no efficacious biologicallybased treatments for these more aggressive forms of breast cancer whose survival rates are less than $26 \%$. Polyamines 
Figure 1

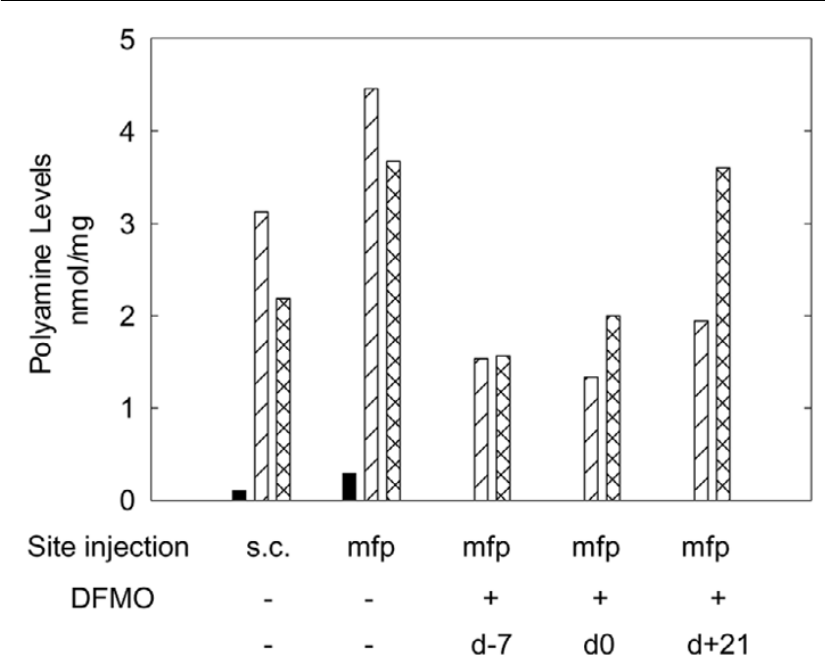

$\alpha$-Difluoromethylornithine (DFMO) (2\% per orally in drinking water) decreases polyamine levels in mammary fat pad tumors of MDA-MB231 cells. Polyamine levels were determined in control subcutaneous (s.c.) and mammary fat pad (mfp) tumors as well as in DFMO-treated tumors grown in the mammary fat pad. DFMO treatment began at either 7 days prior to tumor cell injections (DFMO-7), on the day of injection (DFMO 0) or 21 days post injection (DFMO+21). Putrescine (black), spermidine (diagonal line) and spermine (cross-hatched) levels are graphed as nanomoles per milligram of tumor.

and ODC are increased in breast cancer compared with normal breast tissue, and increased ODC activity directly correlates with a less differentiated and more metastatic tumor phenotype $[5,6,15]$. ODC activity is also an independent adverse prognostic factor for overall breast cancer survival $[5,6,15]$. DFMO, an irreversible inhibitor of ODC, reduces polyamine pools resulting in a cytostatic effect. Our previous studies demonstrated that treatment with DFMO reduces lung metastasis of the hormone-independent MDA-MB-435 breast cancer cell line with only a modest effect on the growth of orthotopic tumors $[8,16]$. This led to two questions: Can inhibition of ODC activity by DFMO decrease metastasis of MDAMB-435 cells to another secondary site, namely bone? Can DFMO decrease orthotopic tumor growth and lung metastasis of another hormone-independent breast cancer cell line, MDAMB-231? DFMO inhibition of tumor growth and metastasis of hormone-independent cells would strongly support targeting the polyamine pathway as a potential treatment.

\section{Polyamine levels are decreased in tumors treated with DFMO}

Polyamine levels were determined in orthotopically (mammary fat pad) and ectopically (subcutaneous) growing tumors in order to demonstrate that DFMO was inhibiting ODC activity. DFMO treatment beginning at either 7 days prior to injection (d-7), on the day of injection (d0) or 21 days following $(d+21)$ injection of MDA-MB-231 cells into the mammary fat pad resulted in decreased putrescine and spermidine levels with only slight effects on spermine levels (Fig. 1). Putrescine levels decreased from an average of $0.200 \mathrm{nmol} / \mathrm{mg}$ tissue in the control samples to below the detection level in all of the DFMO-treated samples, while the spermidine levels decreased from a mean of $3.7905 \mathrm{nmol} / \mathrm{mg}$ in the untreated samples to $1.606 \mathrm{nmol} / \mathrm{mg}$ after treatment. As expected $[17,18]$, the spermine levels decreased only slightly from an average of $2.931 \mathrm{nmol} / \mathrm{mg}$ in the untreated controls to 2.390 $\mathrm{nmol} / \mathrm{mg}$ after treatment. This demonstrates that ODC was effectively inhibited by DFMO treatment.

Curiously, polyamine levels in MDA-MB-231 cells were significantly different when the cells were grown orthotopically versus those grown ectopically (Fig. 1). This result suggested that DFMO treatments might exert different effects on cells depending upon their location. The result is not entirely surprising since several previous experiments have shown that the biologic behavior of tumor cells can vary widely based upon the site of injection [12].

\section{DFMO treatment decreases bone metastasis by MDA- MB-435 cells}

We previously showed that MDA-MB-435 metastasis was decreased by $74 \%$ to the lung when mice were treated with DFMO [9] despite no apparent change in local tumor invasion. That result indicated that DFMO was affecting the later stages of metastasis (i.e. colonization). Breast cancers commonly metastasize to bone [19]. While bone metastases are not directly responsible for most breast cancer deaths, they result in profoundly decreased quality of life. While current therapies exist to decrease osteolysis and reduce sequella of bone metastases, there are few options to diminish bone tumor burden $[19,20]$.

Taking into account the importance of breast cancer metastasis to bone and observations that polyamine levels varied depending upon tumor cell location in the body, we therefore asked whether DFMO had an effect on bone metastasis. To do this, we employed a model recently developed by ourselves using GFP-labeled MDA-MB-435 cells $[10,11]$. This model allows for relatively rapid assessment of the tumor burden in bone and is not dependent solely upon radiographic imaging and histology, which are less sensitive and more laborious, respectively.

As for all xenograft models, metastases in bone are not observed following orthotopic injection; tumor cells are therefore injected directly into the left ventricle of the heart. While metastases develop in other bones, this study involved complete analysis only on femurs because this site reflects changes elsewhere $[10,11]$ and because it is a site commonly affected in women with breast cancer. DFMO treatment was begun 7 days prior to intracardiac injection of GFP-labeled MDA-MB-435 cells and lasted for the duration of the experiment. Mice with fluorescently-labeled femoral tumors were 
(a)

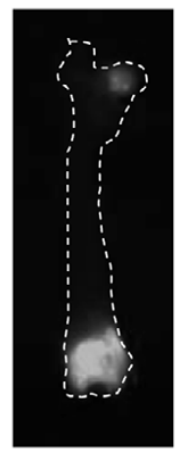

(b)
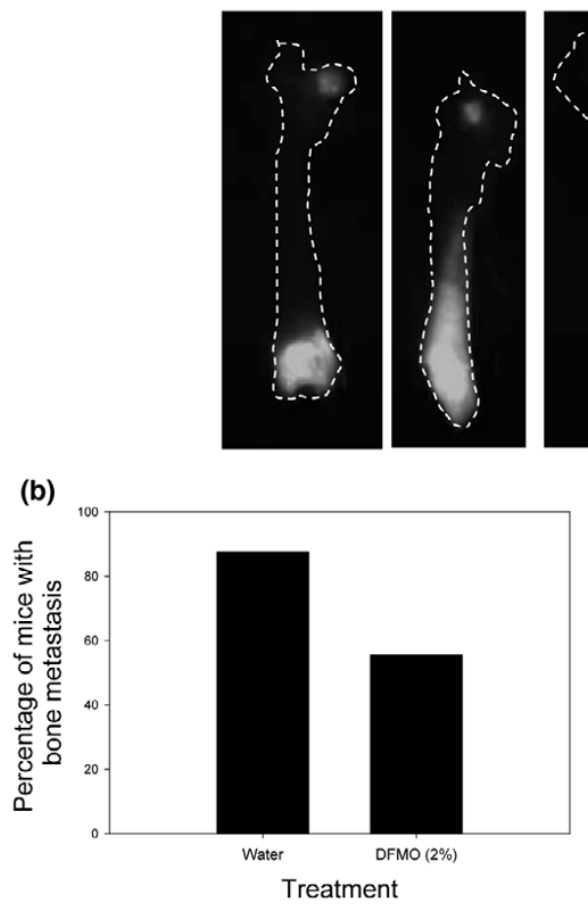

Control
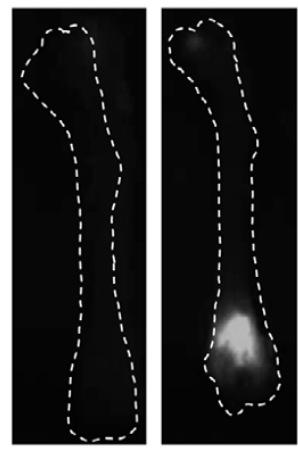

(c)
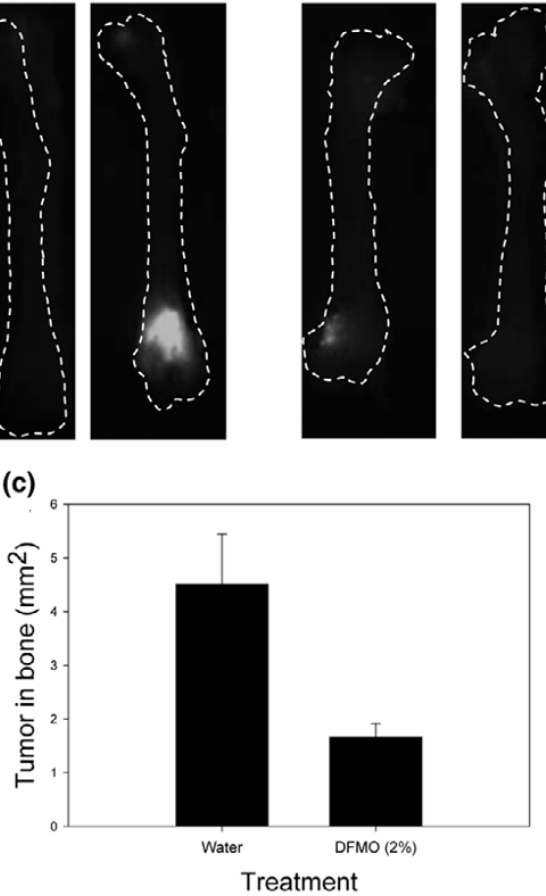

\section{DFMO}
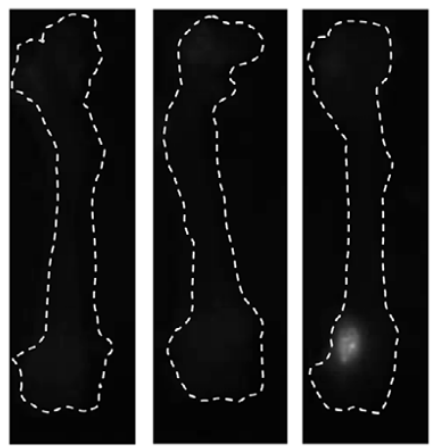

(d)

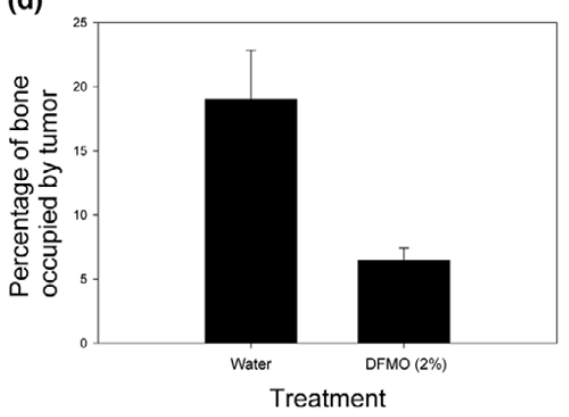

$\alpha$-Difluoromethylornithine (DFMO) decreases the incidence and size of bone metastases from MDA-MB-435 cells. Green fluorescent proteinlabeled MDA-MB-435 cells were injected intracardially into athymic mice. DFMO (2\% per orally in drinking water ad libitum) treatment began 7 days prior to injection and continued for 6 weeks post injection. Bone metastases were visualized using a fluorescence microscope. (a) Photographs of femurs from control $(n=10)$ and DFMO-treated $(n=10)$ mice show the presence and size of the bone metastases. (b) Incidence of metastasis was determined by counting the number of mice with green fluorescence in the femur regardless of size of the mass. Image analysis was used to quantify fluorescence in each bone. (c) The area of tumor in bone was quantified by comparing the total area of the femur with the area containing green fluorescence. (d) The percentage of bone occupied by the tumor was also determined.

counted (Fig. 2a,b). DFMO treatment decreased the number of mice with bone metastases from $87.5 \%$ to $55.5 \%$ (Fig. $2 a, b)$. The GFP label in each femur was then quantified by image analysis and the area of bone occupied by tumor cells was calculated (Fig. 2c,d). DFMO significantly decreased the area of bone occupied by the tumor from 4.51 to $1.69 \mathrm{~mm}^{2}$ (Fig. 2c; $P<0.05$ ) and the percentage of bone occupied by the tumor decreased from $19.03 \%$ to $6.45 \%$ (Fig. 2d; $P<$ 0.05). The intensity of fluorescence was not a variable for these analyses since we previously observed that intensity was dependent upon the depth of tumor cells to the bone surface [10]. As an internal control, however, bones were examined from both sides, and the areas occupied by tumor were found to be comparable (data not shown). Previous and ongoing independent studies have validated tumor location by histology and histomorphometry.

To the best of our knowledge, these are the first data to demonstrate efficacy of DFMO for metastases at a site other than the lung. The polyamine pathway therefore shows promise as a target for decreasing metastasis to multiple sites in breast cancer patients. Our studies have a modest limitation in that they only test efficacy of DFMO against the MDA-MB-435 cells to bone. We and other workers [21-23] have shown that the MDA-MB-231 cells will also colonize bone. But in the absence of GFP-tagged variants, the MDA-MB-231 bone metastasis assays are limited to radiologic or histologic detection methods that are less sensitive or more laborious. They were therefore not utilized here.

\section{MDA-MB-231 orthotopic tumor growth is delayed by DFMO}

This study, combined with previous data, indicates that the polyamine pathway can be modulated to affect primary tumor growth and metastasis of a hormone-independent breast cancer cell line (MDA-MB-435). To begin assessing whether DFMO treatment might be generally efficacious against other hormone-independent breast cancers, we examined orthotopic tumor growth and lung colonization of a second metastatic hormone-independent breast cancer cell line, MDA-MB231.

Orthotopic tumor growth of MDA-MB-435 cells was previously shown to be mildly, but significantly, delayed by or not 
Figure 3

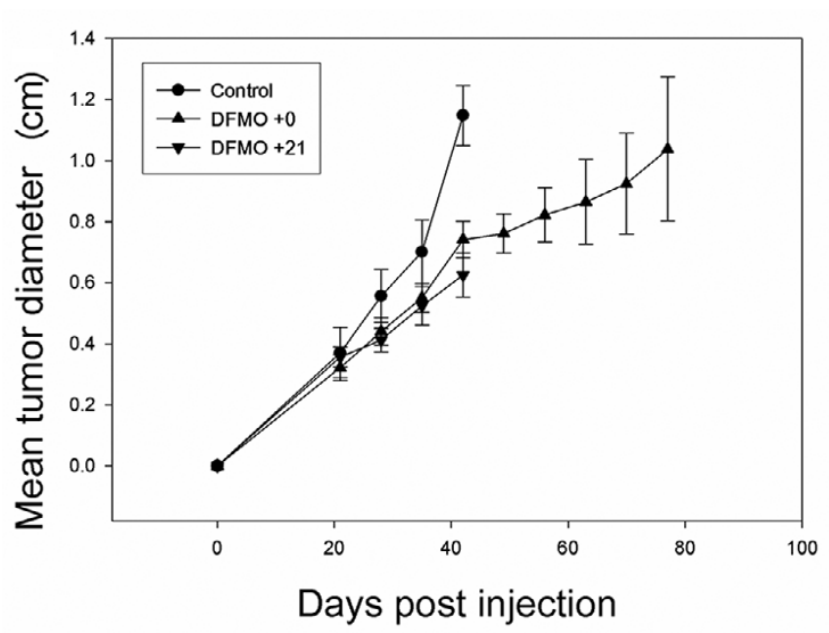

$\alpha$-Difluoromethylornithine (DFMO) delays orthotopic tumor growth of MDA-MB-231 cells. Athymic mice were injected into the second thoracic mammary fat pad with MDA-MB-231 cells. DFMO treatment $(2 \%$ per orally) began either contemporaneously $(\mathbf{\Lambda}, n=20)$ or 21 days post injection $(\boldsymbol{\nabla}, n=3)$. Mammary fat pad tumor length and width were measured once weekly. Control $(\bullet, n=17)$ and 21 days post injection (DFMO+21) animals were sacrificed 6 weeks post injection. Day of injection (DFMO 0$)$ animals were euthanized either 6 weeks $(n=10)$ or 11 weeks $(n=10)$ post injection.

affected by treatment with DFMO $[8,9]$. Untreated MDA-MB435 tumors reached a size of $100 \mathrm{~mm}^{2} 2$ weeks earlier than DFMO-treated tumors. We had also previously shown that MDA-MB-231 orthotopic tumors were delayed by DFMO treatment. We repeated this experiment with slight variations. DFMO treatment began either on the day of injection (d0) or 21 days post injection $(d+21)$ of MDA-MB-231 cells into the second thoracic mammary fat pad and were continued until tumors reached a mean tumor diameter of approximately 1.2 $\mathrm{cm}$ (Experiment 1) or until 6 weeks post injection (Experiment 2). DFMO treatment delayed orthotopic tumor growth to $\sim 1.2$ $\mathrm{cm}$ from 42 days in control animals to 77 days (Fig. 3). At 42 days post injection, control tumors were $1.15 \mathrm{~cm}$ while DFMO-treated tumors were $0.75 \mathrm{~cm}$ when treatment began concurrent with tumor injection, and surprisingly smaller (0.62 $\mathrm{cm}$ ) when treatment began on day 21 (Fig. 3).

DFMO treatment can thus delay orthotopic tumor growth of more than one breast cancer cell line, albeit with heterogeneous responses. MDA-MB-231 cells were more sensitive to DFMO at the primary site; that is, MDA-MB-435 tumors were mildly delayed ( 2 weeks) or not delayed at all compared with a delay of $\sim 5$ weeks for MDA-MB-231. Postponement of DFMO treatment until 3 weeks post injection also inhibited MDA-MB-231 tumor growth, suggesting that DFMO could still be efficacious if administered at the time of diagnosis.
MDA-MB-231 lung metastasis is decreased by DFMO

Like MDA-MB-435 cells, DFMO reduced lung metastasis of MDA-MB-231 cells. The latter, however, had to be evaluated following injection of cells directly into the lateral tail vein because the parental cells are not effective at spontaneous metastasis. This experimental design afforded examination of scheduling since all tumor cells were administered as a bolus. While lung metastasis was reduced in all treatment groups, we were surprised by the pattern of suppression, even though it was reproducible. The incidence of metastases (i.e. proportion of mice developing lung metastases) decreased as expected. Earlier treatment resulted in fewer mice with metastases: $25 \%$, $10 \%$ and $0 \%$ decreased for treatments beginning $d-7, d 0$ and $d+14$ (Fig. 4a). However, the mice receiving DFMO beginning on $d+14$ showed diminishment of the number of metastases per lung ( $42 \%$, mean $=12.5 /$ lung vs $22.2 /$ lung for controls), compared with no effect for $d+7$ (mean $=20.5 /$ lung) and $32 \%$ for d-7 (mean = 15.1/lung) treatment schedules (Fig. 4b). Despite the trend toward decreased lung metastasis, and in contrast to MDA-MB-435 cells, the reductions in lung metastasis never reached statistical significance $(P<0.05)$ for MDA-MB-231 cells. Nonetheless, DFMO was clearly exerting reproducible effects on breast carcinoma growth in the mammary fat pad and in the lung.

\section{DFMO treatment decreases proliferation in MDA-MB- 231 orthotopic tumors}

To begin addressing mechanisms for increased susceptibility of MDA-MB-435 cells to spontaneous metastasis suppression and of MDA-MB-231 cells to suppression of tumor growth, we performed additional studies. In vitro growth was not significantly affected (data not shown). To further assess growth and apoptosis, we examined MDA-MB-231 tumors growing orthotopically by BrdU and TUNEL staining to determine whether DFMO treatment altered proliferation or apoptosis, respectively. We found that DFMO treatment beginning $\mathrm{d} 0$ increased apoptosis (Fig. 5a) and slightly decreased proliferation (Fig. 5b) compared with control tumors. The decrease in BrdU labeling is consistent with, but less dramatic than, the $60 \%$ decrease of Ki67 labeling observed in DFMO-treated MDAMB-435 tumors, while the increase in TUNEL staining is in contrast to the MDA-MB-435 tumors where no change in apoptosis was observed, even though cleaved caspase-3 levels were increased fourfold. Non-apoptotic necrosis was decreased by $60 \%$ in the MDA-MB-435 tumors, resulting in no change in overall tumor volume [24].

\section{Meprin $\alpha$ expression is affected by DFMO in MDA-MB- 435 cells}

Since we previously showed that DFMO does not affect local tumor invasion of either MDA-MB-231 or MDA-MB-435 cells, we reasoned that DFMO was exerting its major effect on later stages of metastasis. This conclusion incorrectly led us to hypothesize that proteinases were not (or only modestly) affected by DFMO treatment. However, recent evidence 
Figure 4
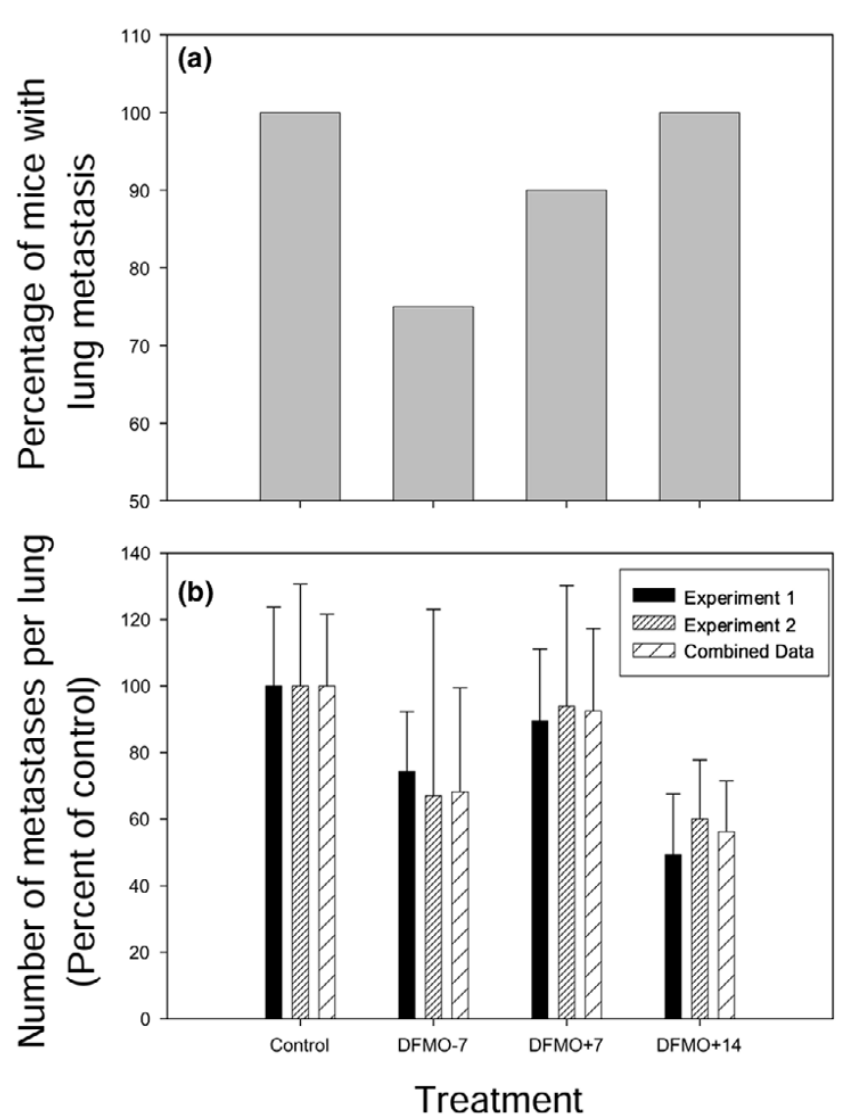

$\alpha$-Difluoromethylornithine (DFMO) treatment decreases lung metastasis of MDA-MB-231 cells. MDA-MB-231 cells were injected into the tail vein of athymic mice. DFMO treatment (2\% per orally) began either 7 days prior to (DFMO-7), or 7 days (DFMO+7) or 14 days (DMFO+14) after, tumor cell injection. Mice were killed either 4 weeks (Experiment 1) or 6 weeks post injection (Experiment 2). Lungs were removed and fixed in Bouin's fixative diluted 1:5 in neutral buffered formalin. Each lung was examined for the presence of surface metastases. (a) The incidence of mice with lung metastases was graphed as a percentage of the total number of mice ( $n=10$ for each group). (b) The average number of metastases per lung was graphed as a percentage of the control ( $n=10$ for each group). Experiment 1 (black) and Experiment 2 (small hatched) are graphed separately as well as combined (large hatched).

suggested that proteinases, and their corresponding inhibitors, have roles in tumor progression at steps other than invasion [25], prompting a closer look.

Meprin $\alpha$ is a zinc-dependent endopeptidase that is normally expressed in kidney and intestinal epithelial cells. It is found as either an apical membrane-bound form or as a secreted protein. At different stages of development, meprin $\alpha$ is expressed in a variety of tissues. In vitro, matrix proteins such as fibronectin, laminin and collagen are meprin $\alpha$ substrates. In some epithelial carcinomas, meprin $\alpha$ secretion can occur both apically and basolaterally, and is activated by plasmin released by stro-
Figure 5
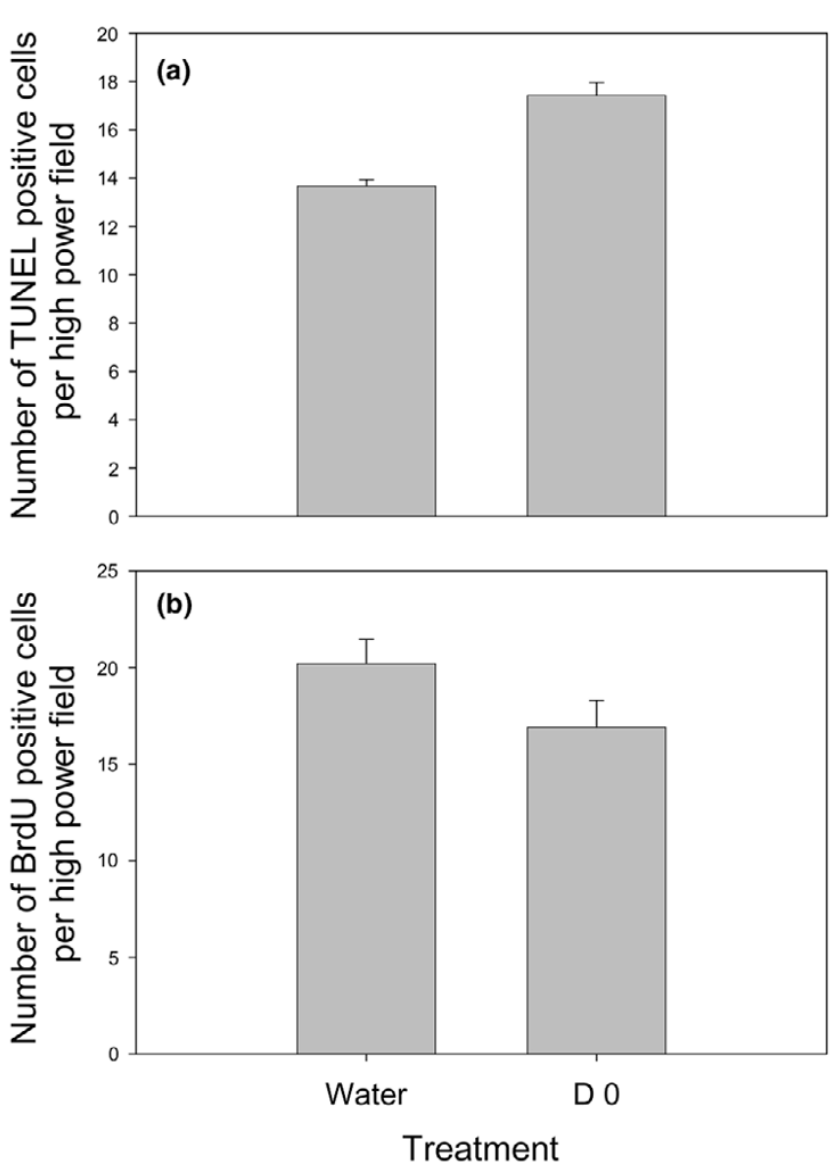

$\alpha$-Difluoromethylornithine (DFMO) treatment affects both cell proliferation and cell death in MDA-MB-231 orthotopic tumors. (a) TUNEL analysis and (b) BrdU analysis of MDA-MB-231 orthotopic tumors from either control mice or mice treated with $2 \%$ DFMO in the drinking water beginning at the day of injection.

mal fibroblasts. Together these data indicate that meprin $\alpha$ can contribute to cancer invasion and/or metastasis.

To assess the impact of DFMO treatment on meprin $\alpha$, MDAMB-435 and MDA-MB-231 cells were treated in vitro with 1 $\mathrm{mM} \mathrm{DFMO} \pm 2.5 \mathrm{mM}$ putrescine (to demonstrate that the inhibition is specific for ODC) for 48 hours. Semi-quantitative RTPCR showed that DFMO treatment had no effect on meprin $\alpha$ expression in MDA-MB-231 cells, while meprin $\alpha$ expression was decreased by greater than $50 \%$ in MDA-MB-435 cells (Fig. 6). Administration of putrescine partially reversed the effect of DFMO in MDA-MB-435 cells (Fig. 6b,d). Although not an exhaustive study, the meprin $\alpha$ results highlight the heterogeneity between different hormone-independent breast carcinomas with regard to their response to DFMO.

While there are likely to be other mechanisms involved, the difference in meprin $\alpha$ levels in DFMO-treated MDA-MB-231 and MDA-MB-435 cells provides at least a partial explanation for 
(a)
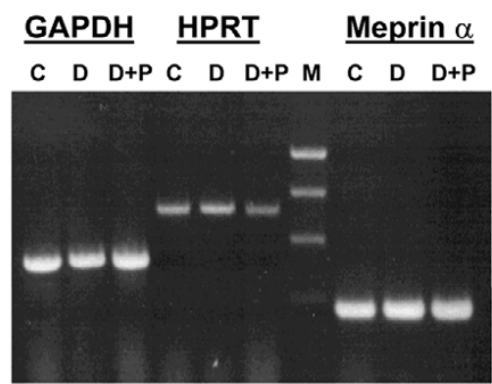

(c)

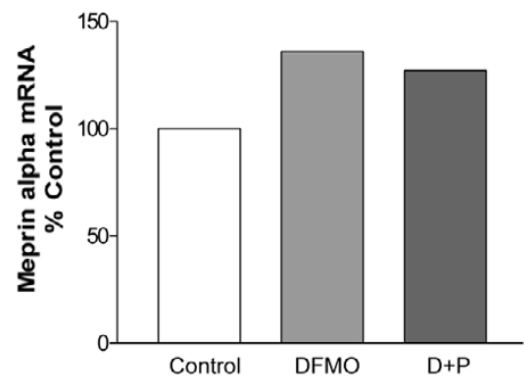

(b)

MDA-MB-435

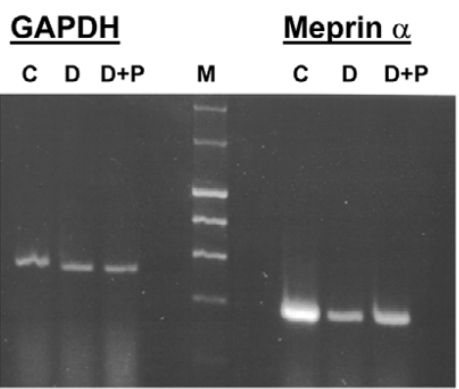

(d)

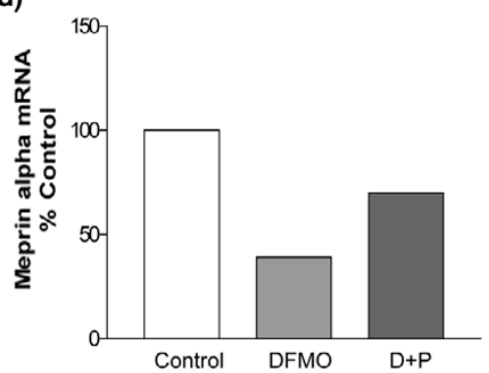

In vitro $\alpha$-difluoromethylornithine (DFMO) treatment decreases meprin $\alpha$ expression in MDA-MB-435 cells, but not in MDA-MB-231 cells. End-point RT-PCR analysis of total RNA from (a) MDA-MB-231 cells or (b) MDA-MB-435 cells, either left untreated (C), treated with 1 mM DFMO for 48 hours (D) or treated with $1 \mathrm{mM}$ DFMO plus $2.5 \mathrm{mM}$ putrescine for 48 hours (D + P). Quantities of meprin $\alpha$ mRNA in (c) MDA-MB-231 cells or (d) MDA-MB-435 cells were normalized to glyceraldehyde-3-phosphate dehydrogenase levels (GADPH) - normalization to hypoxanthine phosphoribosyl transferase [HPRT] was also performed in (a). Meprin $\alpha$ expression levels are graphed as a percentage of control.

the different metastatic phenotypes of these cells in response to DFMO.

In support of our data, another inhibitor of polyamine pathway activity, SL11144, inhibited the growth of both MDA-MB-231 and MDA-MB-435 cells in vitro and of MDA-MB-231 orthotopic tumor growth in vivo [26]. In vitro cell death assays demonstrated a differential effect of SL11144 inhibition on the time line of apoptosis. Specifically, MDA-MB-435 cells exhibited significant DNA laddering at 12 hours after treatment, while MDA-MB-231 cells only showed the initial stages of laddering at 96 hours post-treatment. These results were explained by more potent activation of caspases and rapid cytochrome $c$ release in MDA-MB-435 cells in response to the inhibitor compared with MDA-MB-231 cells. Similar to the studies reported here, SL11144 was effective against both cell lines, but the effect was different between the two lines.

Although heterogeneous responses were observed in the cell lines examined in the present study, the results highlight the potential utility of DFMO for the treatment of advanced hormone-independent breast cancers. Moreover, the finding that DFMO can significantly reduce bone metastasis warrants further studies into the utilization of polyamine pathway modula- tors in combination with other therapies for control of cancer metastasis.

\section{Conclusion}

DFMO, a potent inhibitor of polyamine metabolism, blocks primary tumor growth and/or metastasis to the lung and bone from hormone-independent breast carcinoma xenografts. While the effects are heterogeneous, the findings warrant follow-up into the utilization of polyamine pathway modulators in combination with other therapies for control of this particularly difficult-to-treat class of breast carcinomas.

\section{Competing interests}

The authors declare that they have no competing interests.

\section{Authors' contributions}

MMR provided Figs 1, 3, 4 and 5, and drafted the manuscript. PAP and DJD provided Fig. 2. GM and JSB helped conceive of the meprin part of the study and provided Fig. 6. SW assisted with multiple technical aspects of this study. LMD performed the high-pressure liquid chromatography to determine polyamine levels. AM, MMR and DRW conceived of the study and helped to draft the manuscript. All authors have read and approved the final version of this manuscript. 


\section{Acknowledgements}

The authors would like to thank Dr Jim Griffith, Dr Michael Verderame, Dr Andra Frost and members of the Welch laboratory for helpful discussions and critical reading of this manuscript. We also thank Dr. Lalita Shevde-Samant for assistance with some of the early animal studies. This work was supported by The National Foundation for Cancer Research, DAMD-17-02-1-0541, CA-89019 and CA-87728 to DRW, by DK19691 to JSB and by CA-98237 to AM. This work was also supported by Pennsylvania State University College of Medicine Tobacco Settlement Fund Awards.

\section{References}

1. Gerner EW, Meyskens FL: Polyamines and cancer: old molecules, new understanding. Nat Rev Cancer 2004, 4:781-792.

2. Pendeville H, Carpino N, Marine JC, Takahashi Y, Muller M, Martial $\mathrm{JA}$, Cleveland JL: The ornithine decarboxylase gene is essential for cell survival during early murine development. Molec Cell Biol 2001, 21:6549-6558.

3. Manni A, Wechter R, Verderame MF, Mauger D: Cooperativity between the polyamine pathway and HER-2neu in transformation of human mammary epithelial cells in culture: role of the MAPK pathway. Int J Cancer 1998, 76:563-570.

4. Manni A, Wechter R, Gilmour S, Verderame MF, Mauger D, Demers LM: Ornithine decarboxylase over-expression stimulates mitogen-activated protein kinase and anchorage-independent growth of human breast epithelial cells. Int J Cancer 1997, 70:175-182.

5. Glikman P, Vegh I, Pollina MA, Mosto AH, Levy CM: Ornithine decarboxylase activity, prolactin blood levels, and estradiol and progesterone receptors in human breast cancer. Cancer 1987, 60:2237-2243.

6. Canizares F, Salinas J, de las HM, Diaz J, Tovar I, Martinez P, Penafiel R: Prognostic value of ornithine decarboxylase and polyamines in human breast cancer: correlation with clinicopathologic parameters. Clin Cancer Res 1999, 5:2035-2041.

7. Manni A, Mauger D, Gimotty P, Badger B: Prognostic influence on survival of increased ornithine decarboxylase activity in human breast cancer. Clin Cancer Res 1996, 2:1901-1906.

8. Manni A, Washington S, Craig L, Cloud M, Griffith JW, Verderame MF, Texter LJ, Mauger D, Demers LM, Harms JF, et al.: Effects of alpha-difluoromethylornithine on local recurrence and pulmonary metastasis from MDA-MB-435 breast cancer xenografts in nude mice. Clin Exp Metastasis 2003, 20:321-325.

9. Manni A, Washington S, Griffith JW, Verderame MF, Mauger D, Demers LM, Samant RS, Welch DR: Influence of polyamines on in vitro and in vivo features of aggressive and metastatic behavior by human breast cancer cells. Clin Exp Metastasis 2002, 19:95-105.

10. Harms JF, Welch DR: MDA-MB-435 human breast carcinoma metastasis to bone. Clin Exp Metastasis 2003, 20:327-334.

11. Harms JF, Welch DR, Samant RS, Shevde LA, Miele ME, Babu GR, Goldberg SF, Gilman VR, Sosnowski DM, Campo DA, et al.: A small molecule antagonist of the alpha-v, beta-3 integrin suppresses MDA-MB-435 skeletal metastasis. Clin Exp Metastasis 2004, 21:119-128.

12. Welch DR: Technical considerations for studying cancer metastasis in vivo. Clin Exp Metastasis 1997, 15:272-306.

13. Manni A, Fischer S, Franks M, Washington S, De AR, Griffith J, Demers L, Verderame M, Leiby B, Mauger D: S-adenosylmethionine decarboxylase overexpression reduces invasiveness and tumorigenicity in nude mice of MCF-7 breast cancer cells. Int J Oncol 2001, 19:317-323.

14. Guo YJ, Cleveland JL, O'Brien TG: Haploinsufficiency for Odc modifies mouse skin tumor susceptibility. Cancer Res 2005, 65:1146-1149.

15. Manni A, Grove R, Kunselman S, Aldaz M: Involvement of the polyamine pathway in breast cancer progression. Cancer Lett 1995, 92:49-57.

16. Manni A, Washington S, Mauger D, Hackett DA, Verderame MF: Cellular mechanisms mediating the anti-invasive properties of the ornithine decarboxylase inhibitor $\alpha$-difluoromethylornithine (DFMO) in human breast cancer cells. Clin Exp Metastasis 2004, 21:461-467.
17. Gerner EW, Mamont PS: Restoration of the polyamine contents in rat hepatoma tissue-culture cells after inhibition of polyamine biosynthesis. Relationship with cell proliferation. Eur J Biochem 1986, 156:31-35.

18. Meyskens FL Jr, Gerner EW, Emerson S, Pelot D, Durbin T, Doyle $\mathrm{K}$, Lagerberg W: Effect of alpha-difluoromethylornithine on rectal mucosal levels of polyamines in a randomized, doubleblinded trial for colon cancer prevention. J Natl Cancer Inst 1998, 90:1212-1218.

19. Roodman GD: Mechanisms of disease: mechanisms of bone metastasis. N Engl J Med 2004, 350:1655-1664.

20. Mundy GR: Metastasis: metastasis to bone: causes, consequences and therapeutic opportunities. Nat Rev Cancer 2002, 2:584-593.

21. Kang YB, Siegel PM, Shu WP, Drobnjak M, Kakonen SM, CordónCardo C, Guise TA, Massagué J: A multigenic program mediating breast cancer metastasis to bone. Cancer Cell 2003, 3:537-549.

22. Guise TA: Parathyroid hormone-related protein and bone metastases. Cancer 1997, 80:1572-1580.

23. Yoneda T, Sasaki A, Mundy GR: Osteolytic bone metastasis in breast cancer. Breast Cancer Res Treat 1994, 32:73-84.

24. Matters GL, Manni A, Bond JS: Inhibitors of polyamine synthesis decrease the expression of the metalloproteases meprin alpha and MMP-7 in hormone-independent human breast cancer cells. Clin Exptl Metastasis in press.

25. Chambers AF, Matrisian LM: Changing views of the role of matrix metalloproteinases in metastasis. I Natl Cancer Inst 1997, 89:1260-1270.

26. Huang Y, Hager ER, Phillips DL, Dunn VR, Hacker A, Frydman B, Kink JA, Valasinas AL, Reddy VK, Marton LJ, et al.: A novel polyamine analog inhibits growth and induces apoptosis in human breast cancer cells. Clin Cancer Res 2003, 9:2769-2777 